\title{
The Study Status and Development of Metamaterial Absorber
}

\author{
Wenwen Liu \\ Department of Electronic Engineering \\ Tianjin University of Technology and Education \\ Tianjin, China \\ liu_wen_wen@163.com
}

\begin{abstract}
Metamaterial with superior physical properties is a new type of artificial electromagnetic material. Absorbing material is used widely on stealth weapons of aircraft, missiles, ships, tanks and other more extensive application. the improvement in performance of the metamaterial absorbing body can design new unit structure, design and research the optimized algorithm, use new materials and hybrid materials, explore and combine with new concept, etc. Firstly introduces the working principle of absorbing material and the research progress of absorbing materials and terahertz metamaterials. Finally, the research status of the metamaterial absorber is summarized at the domestic and foreign.
\end{abstract}

Keywords- Physical Properties; Electromagnetic; Metamaterial; Absorber; Terahertz

\section{INTRODUCTION}

Metamaterials usually refers to the Artificial Microstructures of composite materials, and it has extraordinary physical properties which natural materials do not have ${ }^{[1-3]}$. The behavior of metamaterials depends mainly on the artificial structure design.With the progress of electronic technology and the rapid development of the information society, the problem of the interference of the electromagnetic radiation has became a hot research is sue in the world. The metamaterials have become a hot spot of research in the field of physics and materials science because it exhibits extraordinary electromagnetic properties. It has a good application prospect in the terahertz and optics ${ }^{[4,5]}$.

At present, the research direction of metamaterials mainly focuses on: a. the new metamaterial and its design of function, optimization of performance, simulation algorithm; b. the manufacturing device: device fabrication requires high technical level in optical frequency band, because of the sub wavelength size constraints ${ }^{[6-7]}$; c. study on the interaction: the majority of the properties of metamaterials related to the surface / interface waves. In order to further explore coupling of the near field wave and electromagnetic wave in the free space ${ }^{[8]}$. The hotspot applications of metamaterials are as follows: using metamaterials and its characteristics to design and product the various kinds of metamaterial absorbers. Through the metamaterial structure make it realized perfect stealth metamaterials which is invisibility cloak. The metamaterial is applied to microwave circuit or antenna to improve its performance.

Materials mainly focus on the following aspects: a. To develop the materials which can be strong absorbed. Strong absorption is still the main objective of absorbing material pursuit, and it is the most basic requirements of absorbing materials; $b$. To develop the broadband absorbing materials which can compatible multi bands such as the metric, millimeter, infrared wave and so on ${ }^{[9,10]}$. c. To develop the absorbing materials of light weight, thin thickness and which do not affect the flight performance. d. To develop the absorbing materials which have high maintainability, long service life, and ability to adapt to the complex environment.

\section{ABSORPTION MECHANISM}

Absorbing materials is that the electromagnetic wave which project onto its surface is absorbed and attenated. And it makes the electromagnetic energy consumed by converting into heat energy or makes the electromagnetic energy disappeared with interference. Absorbing materials can effectively absorb the incident electromagnetic wave, and make the intensity of target echo attenuated significantly ${ }^{[11]}$.

The absorbing rate is an important parameter of microwave absorbing materials. Absorbing rate is higher and absorbing bandwidth is wider shows that absorption performance is the better.

For a given absorbing material, the absorbing properties are decided by electromagnetic wave transfer rate $T(\omega)$ and electromagnetic wave reflectivity $R(\omega)$. Therefore, the absorbing rate $A(\omega)$ can be represented by the followig formula:

$$
A(\omega)=1-T(\omega)-R(\omega)
$$

From the above formula shows that the absorbing rate is not only related to the reflectivity, also related to the transfer rate. Consider transfer coefficient $S_{21}$ and reflection coefficients $S_{11}$, it can be written as

$$
A(\omega)=1-\left|S_{21}\right|^{2}-\left|S_{11}\right|^{2}
$$

$S_{21}, S_{11}$ are the amplitude of transmission parameters and reflection parameters, respectively.In order to achieve a high absorbing rate, $S_{11}, S_{21}$ must be proper regulated. According to the formula of Smith' parameter inversion:

$$
S_{21}=\left[\sin (n k d)-\frac{j}{2}\left(Z+\frac{1}{Z}\right) \cos (n k d)\right]^{-1} e^{-j k d}
$$

The definition of complex refractive index $\mathrm{n}(\omega)=n^{\prime}(\omega)+j n^{\prime \prime}(\omega)$ and complex impedance 
index $Z(\omega)=Z^{\prime}(\omega)+j Z^{\prime \prime}(\omega), d$ which is medium plate thickness is constant, where

$$
\mathrm{n}(\omega)=\sqrt{\varepsilon(\omega) \mu(\omega)}, \mathrm{Z}(\omega)=\sqrt{\mu(\omega) / \varepsilon(\omega)}
$$

$\mathrm{k}=\mathrm{w} / \mathrm{c}, c$ is the speed of light in vacuum. When $Z$ approaches 1 , realizing impedance matching with free space, the reflectivity is decreased to zero. At this point, the transmission rate is decided by refraction coefficient only, namely:

$$
S_{21}=[\sin (n k d)-j \cos (n k d)]^{-1} e^{-j k d}
$$

According to the Euler's formula, it can be transformed to the:

$$
S_{21}=e^{j(n-1) k d}=e^{j\left(n^{\prime}-1\right) k d} e^{-n^{\prime \prime} k d}
$$

Therefore, the electromagnetic wave transmission rate:

$$
T=\left|S_{21}\right|^{2}=e^{-2 n " k d}
$$

Transmission rate nly concerned with the $n$ ", so when $\mathrm{n}$ " tends to infinity, there is:

$$
\lim _{n^{\prime \prime} \rightarrow \infty} T=\lim _{n^{\prime \prime} \rightarrow \infty} e^{-2 n^{\prime \prime} k d}=0
$$

From the above equation, under the situation of absorbing metamaterial and free space to achieve impedance matching, the magnetic loss and dielectric loss of metamaterials is only related with the $n$ ". This shows that $n^{\prime \prime}$ is the main parameter in the characterization of $\operatorname{loss}^{[12]}$. In addition, according to the above formula, it can explain that the electromagnetic wave of wave vector for $\mathrm{k}$ through the substrate, the overall loss of the electromagnetic wave in the substrate is decided by the thickness for $\mathrm{d}$ of the substrate. Therefore, in order to realize the high absorption of the incident wave, mainly in the impedance matching condition, the $n^{\prime \prime}$ as much as possible to reach the maximum. In order to make the $n^{\prime \prime}$ as large as possible, the substrate should be as thin as possible However, the thickness of the substrate will affect the impedance matching. Therefore, the value of $n^{\prime \prime}$ and perfect the impedance matching contradict each other. Therefore, the thickness of the substrate should be reasonable regulation. Then the $n^{\prime \prime}$ value and the impedance matching achieve a perfect balance, in order to achieve the high absorption of the incident electromagnetic wave.

In short, in order to achieve good absorbing requires two conditions: a. the incident electromagnetic waves as much as possible into the absorbing material without being reflected. $b$. The electromagnetic waves can be attenuated and absorbed by the absorbing material.

\section{MET AMATERIAL ABSORBER ST ATUS}

Domestic and foreign researches on metamaterials absorbing started relatively late, but it has grown by leaps and bounds. Metamaterials absorbing body has the development from the microwave frequencies to $\mathrm{THz}$, infrared and visible light band and from a single band to broadband, double band and multiband, etc.

\section{A. Metamaterial absorber consists of a resonant ring and wire or film}

In 2008, Landy first proposed an absorber, which is composed of elctric ring resonator and short wire structures [13]. As shown in the Fig. 1, the metamaterial absorber was first proposed. Through theoretical and experimental research, the structure of metamaterial absorber can achieve nearly $100 \%$ absorption in the $11.65 \mathrm{GHz}$ for incident electromagnetic wave. This structure provides new ideas and methods for the design of absorber and has been widely concerned. Since then, in the $\mathrm{GHz}$ band and $\mathrm{THz}$ band has new structures proposed.
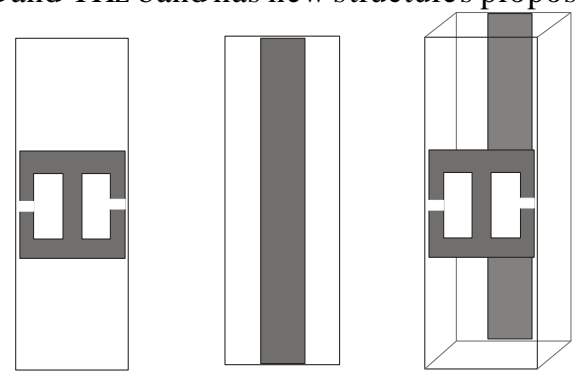

Figure 1. The schematic of unit structure

In 2011, $\mathrm{Hu}$ and liu ${ }^{[14,15]}$ design the strong resonance absorbing metamaterials. Its structure is composed of electric resonators and short wires as shown in Fig.2. Through the theoretical research and simulation, reflected parameter $S_{11}$ in the $10.2 \mathrm{GHz}$ appeared a reflection wave trough, achieving minimum value of $S_{11}=0.11$. The reflectance $R(\omega)$ was about $1.2 \%$ and the transmission rate of $T(\omega)$ is less than $7.5 \%$ in the whole simulation frequency. After calculating, absorption rate is $91.5 \%$ at 10 GHz. The metamaterial can be flexible to adjust magnetic permeability and dielectric constant and acieve high absorption of the incident electromagnetic wave.
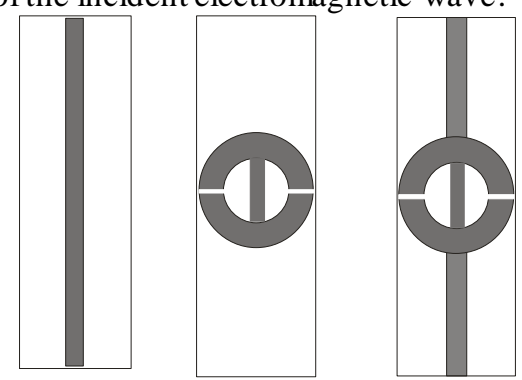

Figure 2. The schematic of structure unit

The above two kinds of absorbing body has a good absorbing performance within the narrow band [16]. It is easy to achieve the regulation of electromagnetic resonance by adjusting the width of the middle rod or short wire. However, absorbing band of absorber is still relatively narrow. It can achieve near perfect absorption only in a specific range, which limits the application of electromagnetic metamaterial absorber in absorption materials. Therefore, the absorbing bandwidth of absorber still needs to continue to expand.

In 2013, Shi Xiaoqiang [17] devised a metamaterial absorber. Its resonant unit was composed of open circle, concentric ring and the metal film as shown in Fig. 3. By electromagnetic simulation software, it's found that the absorption rate of composited absorber (c) is increased, and bandwidth is basically equal to the sum of two absorbing bandwidth of absorber (a) and (b). The vast majority of the electromagnetic wave rips into the internal of the absorber and transmissivity can be thought as zero within the range of frequencies. Through the calculation, 
absorbing rate nearly $100 \%$ achieve perfect absorption at 29.7 GHz. Absorbing bandwidth is $3.1 \mathrm{GHz}$.

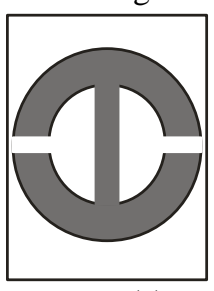

(a)

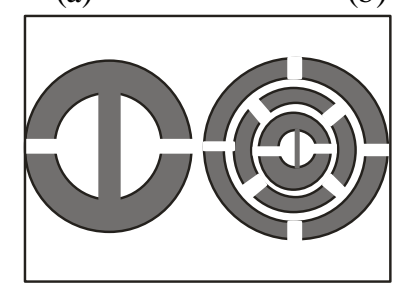

(c)

Figure 3. The schematic of resonant unit

\section{B. Combining algorithm to optimize the metamaterials absorber}

In order to improve the absorption efficiency of metamaterial absorber, unit structure can be optimizated $[18,19]$. SRR unit is widely used in many research fields of electromagnetic metamaterials as the basic unit structure of the electromagnetic metamaterials as shown in Fig. 4.

Huang Wenyuan [20] thought using DES - CACO hybrid algorithm and combining with the electromagnetic simulation software, the return loss and loss tangent value of SRR unit was optimized. By the electromagnetic simulation software simulating the electromagnetic metamaterials structure, structure variables are setted. Then, calculating the $S$ parameters of the structure, combining with VB interface program, mix optimization results as in DES - CACO algorithm of fitness value, to carry on the multiple iterations and to obtaine the optimal structure parameters. Numerical simulation results show that loss tangent value is greatly reduced, almost to zero within the range of the spectrum under the condition of which the structure of the other basic features invariable such as negative refraction, etc.

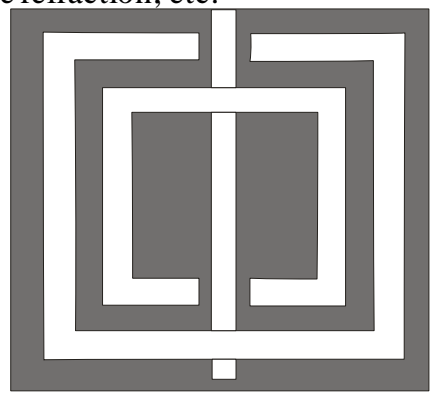

Figure 4. The schematic of SRR structure unit

Huang Wenyuan also optimizes terahertz electromagnetic metamaterials absorber of the type IIIbroadband as shown in Fig. 5 by applying DES - CACO hybrid algorithm, and increases its absorption bandwidth. The simulation results show that the absorption rate of electromagnetic metamaterials is over $90 \%$, and absorption bandwidth increase about $29 \%$ of the original bandwidth in the range of $0.87 \mathrm{THz}$ to $0.97 \mathrm{THz}{ }^{[20]}$.
Due to the limitation of computer software and hardware configuration, the slower rate of optimization algorithm and the set of population size and number of iterations is relatively small, which may make the parameters of the optimized structure fall into the situation of a local optimum. However, the experimental results confirmed that the method of design can effectively reduce the lossy characteristics of the unit structure of electromagnetic metamaterials, and increased the absorption bandwidth of electromagnetic metamaterials greatly. This helps it to be widely applied to the field of electromagnetic metamaterials device used, and expands the new train of thought for the future design and research of electromagnetic metamaterials.

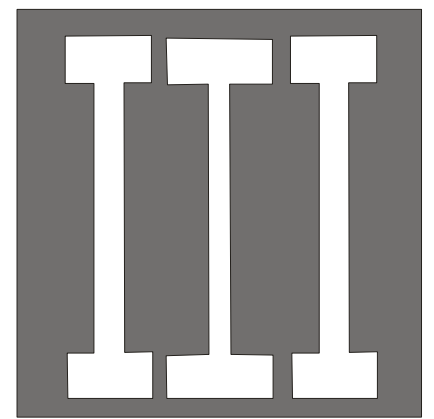

Figure 5. The schematic of III structure unit

\section{Terahertz metamaterials and absorbers}

Graphene is a new type of carbon materials which has good thermal stability and chemical stability ${ }^{[21-23]}$. Li Jiayuan and Zhou Yixuan [8] demonstrated a grapheneSRRs hybrid structure based on a large-area graphene and $\mathrm{N}$-doped graphene synthesized by APCVD. Hybridization of graphene and N-doped graphene with SRRs leads to an intraband carrier coupling between graphene and SRRs in $\mathrm{THz}$ region. They observed maximum enhancement of $33 \%$ in non-resonant region and $24 \%$ in resonant region with the hybridization of graphene and metamaterials in experiment ${ }^{[24]}$.

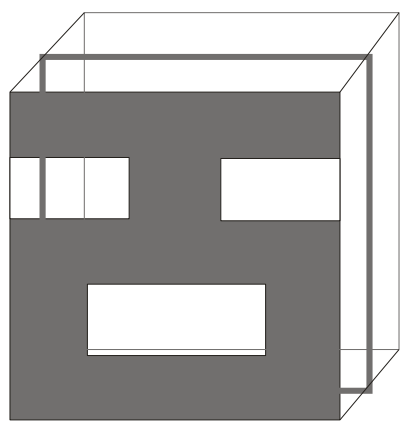

Figure 6. The schematic of structure unit

The results suggest that there exists an exponential relationship between coupling strength and $\mathrm{THz}$ response in both resonant and non-resonant region. Through the experimental study, graphene hybrid materials can make the resonant frequency shift and the coupling strength between the resonant rings. The metamaterial absorbers achieve perfect impedance matching through the ring resonator with free space. Therefore, it can consider the 
technology of the hybrid applys in the design and implementation of the metamaterial absorber in the future.

Despite high absorptivity achieved, most of the existing $\mathrm{THz}$ metamaterial absorbers are polarizationindependent or only sensitive on one direction [25]. However, in some application cases, $\mathrm{THz}$ polarization imaging, selective spectral detection, $\mathrm{THz}$ sensing and polarization multiplexing, high absorption peaks in two orthogonal directions are very useful and demanded. $\mathrm{Hu}$ Fangrong and Zou Taobo fabricated a polarizationdependent terahertz metamaterial absorber as shown in Fig. 6. It is made of a metal film, a dielectric spacer and a metal pattern layer ${ }^{[26]}$.

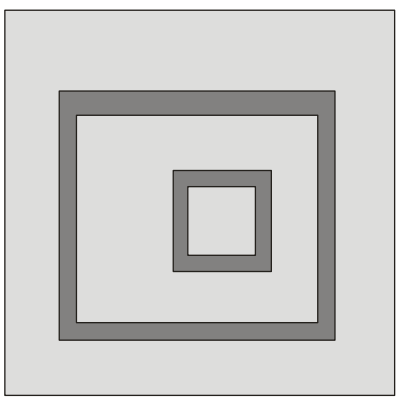

Figure 7. The unit structural schematic

There is a new terahertz broadband metamaterials absorber which is proposed by Wang Benxin and Wang Lingling et.al. Its schematic diagram is shown in Fig. 7. The unit structure is composed of two asymmetric metallic square rings and a metallic ground plane separated by a dielectric layer ${ }^{[8]}$. The broadband terahertz metamaterial absorber is realized by making use of the coupling effect of two asymmetric patterned square rings. The shift of the inner ring along the direction of the incidence electricfield makes its corresponding resonance mode split into two absorption peaks. The absorption of the two resonant bands is greater than $98 \%$. The bandwidth of the two split absorption peaks is adjusted by the coupling distance of the two square rings. The absorber bandwidth can be tuned

The concept of using the coupling effect between rings for absorbing is applicable to other types of asymmetric absorber structure. And it can readily be scaled up to the structures that are working in the microwave frequency range. The proposed metamaterial absorber has potential applications in detection, imaging and stealth technology.

\section{APPLICATION OF ABSORBING MATERIALS}

With the development of the radar detection technology and missile guidance technology, absorbing material on aircraft, missiles, ships, tanks and other more extensive application of stealth weapons. Absorbing material is mainly used in stealth technology. In stealth and electromagnetic compatibility technology, the effect of the electromagnetic absorbing metamaterial is very strong and the position is also very prominent. It is the "secret weapon" in modern military. It is mainly used in stealth technology and improving the machine performance and other aspects.

It is an important means of anti radar detection that the surfaces of the aircraft, tanks, ships, missiles and other important weapons and military equipment are coated of the electromagnetic absorbing materials. Because it not only can be effective absorption will investigate waves, but also can be attenuation reflected signal. Therefore, as to break through the radar sectors, reduce the attack of infrared guidance missile and laser weapon of weapon system. In addition, the body of the plane false intercepted or false tracking by high sensitive airborne radar, due to false signals generated by electromagnetic wave reflection. In order to reduce interference, the foreign countries commonly use the magnetic shielding of absorbing materials to improve the performance of radar. Such as around the fuselage of radar or communications equipment or distractors coated with absorbing material, make it more sensitive and accurate target, so as to improve the quality of communication.

The development of intelligent metamaterials absorber is a potential direction. It can change to absorb frequency and bandwidth, so as to realize efficient absorbing function according to the change of the surrounding environment. The research of the intelligent advantageous is helpful to realize the automatic and intelligent stealth of stealth weapons and to improve the capabilities of stealth weapons and equipment.

\section{CONCLUSIONS}

The design of metamaterials absorber depends on the research of the basis theory and the development of new materials. Improves the performance of the metamaterial absorbing body can design new unit structure, design and research the optimized algorithm, use of new materials and hybrid materials, explore and combined with new concept, etc. With the progress of science and technology and the improvement of people need, the countries all over the world to the performance research of the electromagnetic absorbing material is becoming more and more attention, and obtaine the periodic achievements in the study. At present, the research and development of the electromagnetic absorbing materials is particularly important. Therefore, people should understand and grasp timely the research dynamic and the development direction of absorbing material.

\section{REFERENCES}

[1] X. Chen. Research on the Electromagnetic Characters and Applications of Metamaterial, National University of Defense Technology, Master Degree Thesis, 2013.

[2] J. P. Zhong. Research on key techniques and applications of multiband electromagnetic metamaterials, University of Electronic Science and Technology, Master Degree Thesis, 2013.

[3] X. Wu. Research on Characteristics and Non-linear Fitting of the Electromagnetic Metamaterials Parameters, Nanchang Hangkong University, Master Degree Thesis, 2014.

[4] D. H. Ding. Research Status and Outlook of High Temperature Radar Absorbing Materials, Journal of Inorganic Materials, Vol.29 No.5, May, 2014.

[5] N. Ma. Absorption Characteristics and Structural Design of Infrared Absorbing Metamaterial, Changchun University of Science and Technology, Master Degree Thesis, 2014.

[6] H. Han. Fabrication of Terahertz Metamaterial by Laser Printing and Resonator Research, CHINA JILIANG UNIVERSIT Y, Master Degree Thesis, 2014.

[7] Z. H. Zhou. Design and preparation of a low frequency absorber based on hollowed-out cross-shapedmeta-material structure, Acta Phys. Sin. Vol.63, No.18 (2014) 184101.

[8] B. X. Wang. Tunable bandwidth of the terahertz metamaterial absorber. Optics Communications, 325 (2014) 78-83. 
[9] F. Chen. Design of dual narrow-bands metamaterial absorber, Journal of Guilin University of Electronic Technology, Vol.34, No. 3,2014

[10] X. Z. He. Study on the deesign of an electromagnetic wavw dual band metamaterial-based absorber, Journal of Beijing Information Science and Technology University, Vol.29 No.2, 2014.

[11] S. B. Chen. Current status of THz band metamaterial absorbers, INFORMATION AND ELECTRONIC ENGINEERING, Vo1.9, No.3, pp.270-273, 2011.

[12] Q. X. Gong. Study on absorbing properties and mechanism of cross-shaped metamaterial absorber. Infrared and Laser Engineering, Vol.42 No.6,2013.

[13] Landy N I, Sajuyigbe S, Mock J, et al. Perfect metamaterial absorber, Phys Rev Lett, 2008, 100(20): 1-4.

[14] C. S. Hu. Numerial research on tunable metamaterial absorber, Hubei University of Technology, Master Degree Thesis, 2011.

[15] Y. Y. Dong. Research on properties of metamaterial absorbers in multi-layer configureation, Function Material, Vol 46, No. 5, 015.

[16] L. Y. Liu. Research on Absorbing Properties of Metamaterial Absorber, Materials Review, 2010,24 (010) : 1-3.

[17] X. Q. Shi. Design and Research on a kind of broad-band Metamaterial Absorber, Zhengzhou University, Master Degree Thesis, 2013.
[18] Y. Yang. Study on Metamaterial Absorbers and Optimization, Xidian University, Master Degree Thesis, 2011.

[19] D. Q. Huang. Broadening and Optimizing Microwave AbsorberBandwidth by Metamaterial Unit Contour Method, Journal of Material Engineering, No.11, pp.1-6, 2014.

[20] W. Y. Huang. Optimization Design on Metamaterial Element Loss and THz Absorber, Southwest Jiaotong University, Master Degree Thesis, 2013.

[21] J. Luo. Synthesis and characterization of Fe3o4/graphene composites, Function Material, Vol.46 No.7, 2015.

[22] Y. R. Lai. Preparation and study on microwave absorption properties of $\mathrm{Fe}-\mathrm{Ni} / \mathrm{RGO}$ nanocomposites, J Magn Mater Devices, Vol. 46, No. 2, 2015.

[23] N. Zhang. Research Progress of Graphene Based on Microwave Absorbing Composite Materials, Vol. 29, No. 5, 2014.

[24] J. Y. Li. Graphene-metamaterial hybridization for enhanced terahertzresponse, CARBON, 78 (2014) 102-112.

[25] L. Zhao. Polarization-independent L-shaped perfect metamaterials absorber in mid-infrared region, Harbin Institute of Technology, Master Degree Thesis, 2014.

[26] F. R. Hu. Polarization-dependent terahertz metamaterial absorber with high absorption in two orthogonal directions, Optics Communications, 332 (2014)321-326. 